\title{
Simulation study of cyclic-tethered nanocube self-assemblies: effect of tethered nanocube architectures
}

\author{
X Zhang ${ }^{1}$, Z L Zhang ${ }^{2}$ and S C Glotzer ${ }^{1,2}$ \\ ${ }^{1}$ Department of Materials Science and Engineering, University of Michigan, Ann Arbor, \\ MI 48109-2136, USA \\ ${ }^{2}$ Department of Chemical Engineering, University of Michigan, Ann Arbor, MI 48109-2136, \\ USA \\ E-mail:sglotzer@umich.edu
}

Received 7 September 2006, in final form 14 December 2006

Published 7 February 2007

Online at stacks.iop.org/Nano/18/115706

\begin{abstract}
Self-assembly of functionalized nano building blocks (NBBs) is a promising avenue for 'bottom-up' nanomaterials design. Experimental studies on functionalized polyhedral oligomeric silsesquioxane (POSS) nanocubes have revealed a wide variety of nanostructures from their assemblies. Our previous simulation studies have reproduced some of these nanostructures and predicted unusual phase behaviours imparted by the unique geometry of the nanocubes and their close packing patterns. Recent experiments further inspire us to explore the effects of tether topologies on functionalized nanocube self-assemblies. We use a simplified model and perform stochastic molecular dynamics simulations to map the morphological phase diagrams of cyclic tethered nanocubes with varying tether topology, tether number, and tether placement. Our results illustrate that the steric influence of the tethers can be manipulated to confer precise control over the self-assembled nanostructures and the phase behaviour. The novel controlling factors investigated in our study suggest new opportunities in controlling functionalized NBB self-assemblies.
\end{abstract}

(Some figures in this article are in colour only in the electronic version)

\section{Introduction}

Self-assembly of nanoscale molecules and particles into nanostructures is a promising strategy for 'bottom-up' materials design, as the shape and interaction anisotropy of the nanoscale building blocks (NBBs) can be exploited to control the self-assembled morphologies and the resulting nanostructures [1-3]. Functionalized polyhedral oligomeric silsesquioxane (POSS) molecules are among the most studied systems of such NBBs [4-10]. These POSS molecules have a cubic-shaped $\mathrm{Si}_{8} \mathrm{O}_{12}$ framework where the silicon atoms sit at the cube corners and the oxygen atoms are interspersed along the edges. Each corner of the POSS nanocube can be functionalized with a variety of organic groups [11-13] to confer precise control over the geometry of the NBBs and their interactions. Combined with suitable temperature and solvent conditions, POSS molecules of tailored geometry and interactions can selfassemble into a variety of interesting nanostructures. For example, micelles and vesicles are found in self-assembled poly(ethylene oxide) [14] and polyoxazoline [15] tethered POSS molecules in aqueous solutions. Lamellar structures are reported to result from polystyrene tethered POSS molecule assemblies [9]. Zigzag-shaped cylinders or lamellae are observed in polyimide tethered POSS systems [16]. The assembled hybrid POSS-polymer materials have enhanced thermal, mechanical, electronic and dielectric properties compared to conventional neat POSS or polymer materials, and therefore become promising candidate materials for next generation nanodevices [17-19].

The versatility and superb properties of POSS materials demonstrated by experimental works have inspired a number 
of simulation studies on the self-assembly of tethered POSS molecules [20-23]. Lamm et al studied octafunctionalized POSS molecules with lattice Monte Carlo simulations and successfully reproduced network properties observed in experiments [24]. Sheng et al performed Monte Carlo simulations to investigate POSS network topologies [25]. Chan et al developed a 'minimal model' of tetra-tethered POSS nanocubes from $a b$ initio computations and studied the self-assembled phase diagrams with Brownian dynamics simulations [26]. Their simulated phase diagrams predict micelle phases in the low density regime and cylindrical phases in the high density regime. A similar model was also used to study the self-assembled morphologies of mono-tethered POSS molecules [27] and the results suggest that lamellae are the preferred phases in mono-tethered POSS systems under the same solvent conditions as in Chan et al. The different phase behaviour of tetra- and mono-tethered POSS nanocubes suggested that the number of tethers and their placement on the nanocubes can be crucial in controlling the self-assembled morphologies. Here we further investigate the role of the tethers in controlling the self-assembled structures of attractive nanocubes.

Recently, Yank et al [28] showed that attaching cyclic tethers to rods produces novel barrel-like structures previously unknown to rod self-assemblies. Auner et al [29] also demonstrated that it is possible to synthesize cyclic-tethered POSS, and other cyclic-tethered cubes. In this work, we simulate minimal models of cyclic tethered nanocubes with varying tether topology, number and placement and map their morphological phase diagrams. Our results help elucidate the contribution of these novel controlling factors in NBB self-assemblies, and suggest new avenues for bottom-up nanomaterials design.

\section{Model}

As the self-assembly process of functionalized NBBs usually involves thousands of molecules and millions of atoms, atomistic-scale simulations are often prohibitively expensive in terms of their computational cost. Therefore, we adopt a simple 'minimal' or phenomenological model in this study, in which a group of atoms are represented by a single coarsegrained 'bead.' A number of beads are rigidly bonded together to mimic the geometry of the cubes. Previous $a b$ initio computations showed that a POSS cube is reasonably modelled as a rigid cube [26]. A chain of beads are connected with finitely-extensible nonlinear elastic (FENE) springs [30] to represent a polymer tether. Previous studies by us have demonstrated the effectiveness of these models in capturing self-assembly behaviour of 'shape amphiphiles' such as polymer-tethered nanoparticles [31, 32]. Such models have also been used in tethered POSS nanocube simulations [26, 27], where eight POSS beads are connected by rigid bonds to form the cubic inorganic core of POSS molecules, and tether beads are connected by FENE springs to form the organic tethers. FENE springs are also used to attach the tethers to the corners of POSS nanocubes. We use a similar model, shown in figure 1, to investigate functionalized POSS molecules with a variety of tether topologies, numbers

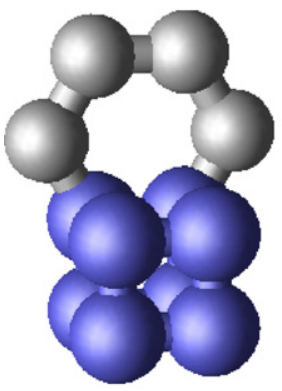

(a) Model system 1

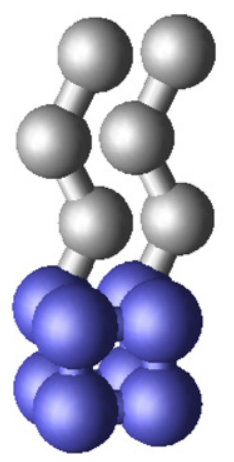

(c) Model system 3

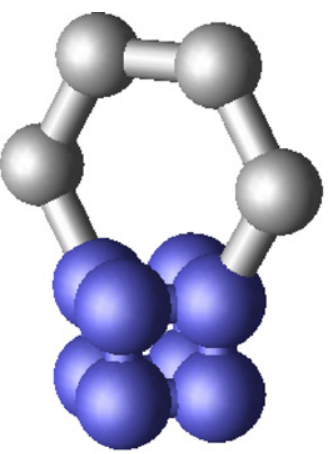

(b) Model system 2

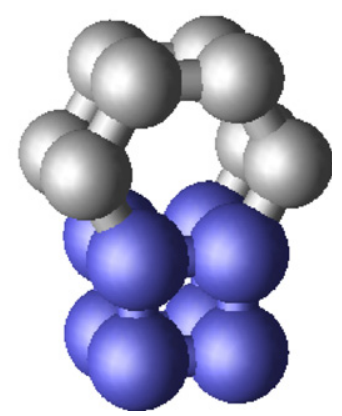

(d) Model system 4
Figure 1. Simplified minimal models of tethered POSS molecules. (a) Model system 1: POSS nanocube with a cyclic tether attached to the cube corners along the same edge. (b) Model system 2: POSS nanocube with a cyclic tether attached to the cube corners of a face diagonal. (c) Model system 3: POSS nanocube with two linear tethers attached to two cube corners along the same edge. (d) Model system 4: POSS nanocube with two cyclic tethers attached to the same face. Here we study all four architectures, each with varying tether length.

and placements. We note that our model is also applicable to tethered nanocrystalline cubes of similar geometry.

The solvent-mediated interactions between tethered NBBs are simulated with conventional non-bonded interaction potentials. In a selective solvent poor for nanocubes and good for tethers, the truncated and shifted Lennard-Jones (LJ) potential is applied between cube beads to mimic their attractive interaction in poor solvent, whereas the WeeksChandler-Andersen (WCA) potential is applied between all other pairs of beads to capture their repulsion in good solvent. This selective solvent condition is applied to all of the simulation systems in this study, as it induces sufficient thermodynamic immiscibility for microphase separation. In addition, we have previously investigated similar solvent conditions using the same model $[26,27]$ and thus we can investigate the novel tethered cube topologies within a wellcontrolled framework. The force parameters in this work are set to the same reduced values as in previous studies [26, 27], i.e. FENE spring constant $\kappa=30.0$, FENE maximum bond length $R_{0}=1.5$, LJ attractive well depth $\varepsilon=1.0$, LJ and WCA sphere diameter $\sigma=1.0$, and interaction cutoff distance $r_{\mathrm{c}}=2.5 \sigma$ for the LJ potential and $r_{\mathrm{c}}=2^{1 / 6} \sigma$ for the WCA potential. 


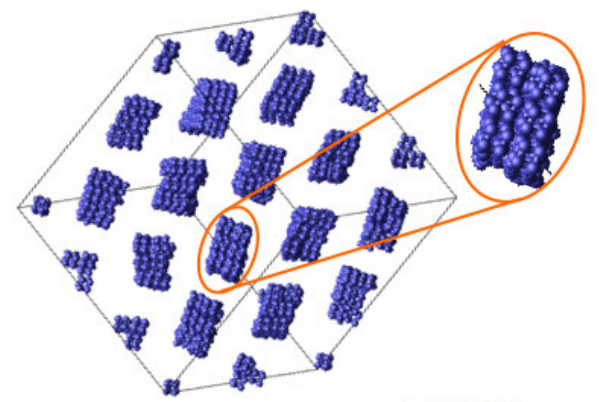

(a)

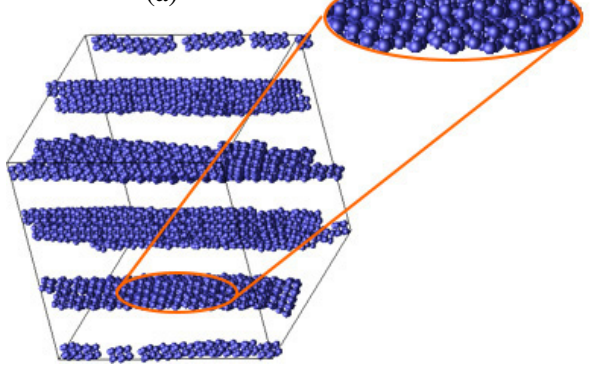

(b)

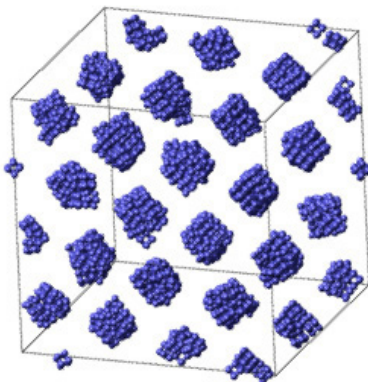

(c)

Figure 2. Typical morphologies from simulation snapshots. (a) Rectangular-shaped cylinders. (b) Lamellae. (c) Square-shaped cylinders. (For clarity, the tethers have been removed and only the POSS nanocubes are shown in the snapshots.) Call-outs show blow-ups of local cube packing.

As in our previous studies, we use the Brownian dynamics simulation $[33,34]$ method-a stochastic molecular dynamics method - and the quaternion algorithm [35] to simulate the 3D movement of tethered nanocubes and to predict the equilibrium structures. Compared with conventional molecular dynamics methods, the method used in this study allows the implicit treatment of solvent molecules and a larger time step; thereby enabling simulations of much larger time and length scales to tackle the problem of functionalized NBB self-assemblies. We use the same parameters as in our previous studies on monoand tetra-tethered nanocubes [26, 27], including a friction coefficient which is set to 1.0 in reduced units. Previous simulations showed that this choice of friction coefficient permits a reasonable choice of time step and does not affect equilibrium morphologies [27a].

To investigate the effects of a wide variety of thermodynamic parameters, including temperature, volume fraction, tether length, tether topology and tether placement, we map two morphological phase diagrams for each of the model systems shown in figure 1 , one with a fixed tether length and varying volume fraction, and the other with a fixed volume fraction and varying tether length. For each point in the phase diagrams, the system is initially relaxed at a sufficiently high temperature to achieve a random, disordered configuration, and then slowly cooled to the target temperature. The equilibrium structure is identified by visual inspection of the simulation snapshot and monitoring of the energy as a function of time. We use several standard ways to make sure that the final states we obtain are indeed equilibrium states, such as varying the temperature path, starting from different initial states, and running multiple independent simulations. We found that the obtained structures are independent of all these variations, as required for equilibrium phases. A typical simulation run of 1000 tethered cubes reaches equilibrium in 10-30 million time steps, with a CPU cost of 200-1000 hours on a $2.0 \mathrm{GHz}$ Apple PowerPC G5 processor (roughly 5.6 microseconds per bead per time step). The combined results presented in this paper required approximately $113000 \mathrm{CPU}$ hours.

\section{Results and discussion}

\subsection{Nanocubes with a cyclic tether attached to the cube corners along the same edge}

We first simulate nanocubes with a single tether attached at both ends to adjacent cube corners (model system 1, as shown in figure 1(a)). We observe both lamellar and cylindrical structures, shown in figures 2(a) and (b). Insets in figures 2(a) and (b) demonstrate that in both lamellar and cylindrical structures the nanocubes are locally packed face-to-face. This packing pattern is also found in simulated mono- and tetratethered POSS systems [26, 27]. As discussed in [27], such cubic face-to-face packing is introduced by the cubic geometry of the nanocubes and their strong attractive interaction in poor solvent at low temperature. Interestingly, we find that the cross-section of the cylinders exhibits a rectangular shape (figure 2(a)), as opposed to cylinders of square crosssection reported for tetra-tethered nanocube systems [26] and cylinders of circular cross-section for surfactant systems. This structural difference indicates not only the favourable faceface packing of nanocubes but also demonstrates how building block topology influences the geometry of the self-assembled nanostructures. We will revisit this in section 3.4 when we discuss nanocubes with two cyclic tethers (model system 4 in figure $1(d))$.

Figure 3(a) shows the phase diagram of model system 1 with a fixed volume fraction $(\phi=0.3)$ and varying tether length. Disordered phases are observed at high temperatures 

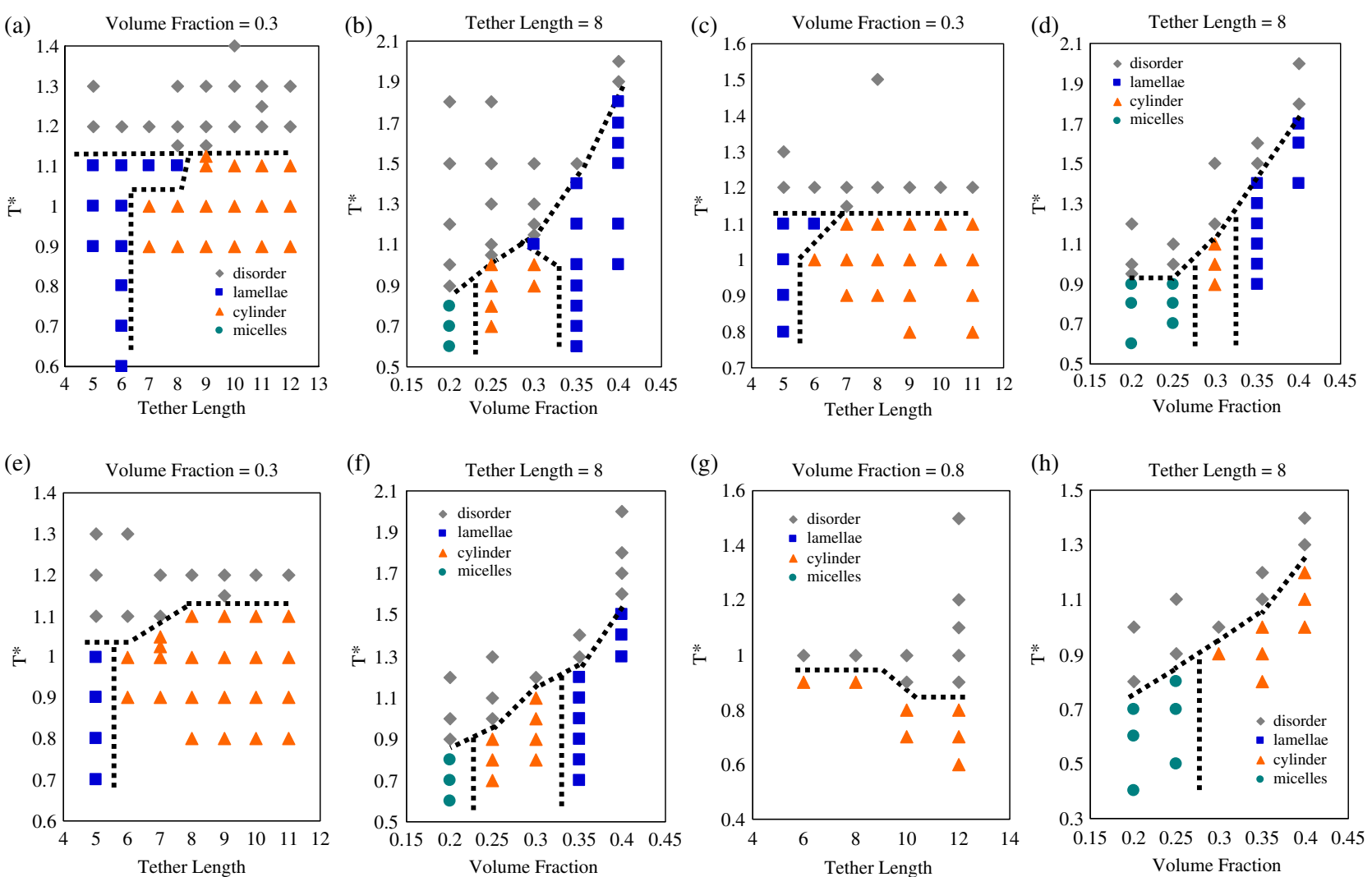

Figure 3. Morphological phase diagrams of tethered POSS nanocubes. (a) Model system 1, with a fixed volume fraction $\phi=0.3$ and varying tether length. (b) Model system 1, with a fixed tether length of eight beads and varying volume fraction. (c) Model system 2, with a fixed volume fraction $\phi=0.3$ and varying tether length. (d) Model system 2, with a fixed tether length of eight beads and varying volume fraction. (e) Model system 3, with a fixed volume fraction $\phi=0.3$ and varying tether length. (f) Model system 3, with a fixed combined tether length of eight beads and varying volume fraction. (g) Model system 4, with a fixed volume fraction $\phi=0.3$ and varying tether length. (h) Model system 4, with a fixed combined tether length of eight beads and varying volume fraction. The grey diamonds represent disordered phases; green circles represent the micelle phase; yellow triangles represent the cylinder phase; and blue squares represent the lamellae phase. The phase boundaries are drawn as guides to the eye.

and ordered lamellar and cylindrical phases are observed at low temperatures. Specifically, lamellar phases are the preferred phases for short tether lengths (five and six beads) and cylindrical phases are the preferred phases for long tether lengths (nine beads and longer). For seven- and eight-bead tethers, lamellar phases are observed at higher temperature $\left(T^{*}=1.1\right)$ and cylindrical phases are observed at lower temperatures $\left(T^{*}=1.0\right.$ and below). Our previous study of mono-tethered cubes predicted that the face-to-face packing of nanocubes in poor solvent favours the flat domain interface in a lamellar structure and the lamellar phases are the only ordered phases observed under such solvent conditions [27]. Here, both lamellar and cylinder phases are found. This behaviour can be explained by the fact that the cyclic tethers have stronger steric effect than linear tethers. Since both ends of the cyclic tethers are attached to the nanocubes, there are more tether beads restricted to the immediate vicinity of nanocubes than in the mono-tethered systems. The tethers are further swollen in good solvent and tend to occupy more volume surrounding the nanocubes, interrupting the long range, flat, face-to-face close packing required by lamellar phases. As tether length increases, the volume occupied by the tethers also increases, favouring cylindrical phases. With intermediate tether lengths, as the energetic term begins to dominate the free energy at low temperatures, the tethers tend to occupy more space to minimize their repulsive interactions, again favouring cylindrical phases.

Figure $3(\mathrm{~b})$ shows the phase diagram of model system 1 with a fixed tether length (eight beads) and varying volume fraction. As in conventional surfactant phase diagrams, micellar structures are found in the low volume fraction regime $(\phi=0.2)$ when the temperature is decreased below the order-disorder transition (ODT) temperature, and the highly ordered cylinder and lamellae phases are found at higher volume fractions $(\phi=0.25$ and above). The trend of the ODT temperature increasing with higher volume fraction is also consistent with typical surfactant phase diagrams. In the highly ordered phase domain, cylinders are the preferred phases with relative low volume fraction $(\phi=0.25$ and 0.3$)$ and lamellae are the preferred phases with relative high volume fraction ( $\phi=0.3$ and above). This also can be explained by the steric influence of repulsive tethers. With a higher volume fraction, the tether beads are forced to occupy less volume, thereby decreasing their disruptive effect on the flat, face-toface cubic packing required for lamellar phases. Conversely, with a lower volume fraction, the tethers have more space to swell, frustrating the flat face-to-face packing of the nanocubes and stabilizing the cylindrical phase. 
3.2. Nanocubes with a cyclic tether attached to the cube corners of a face diagonal

Next we study nanocubes with a tether attached at both ends to the cube corners along a face diagonal (model system 2 as shown in figure 1(b)) and compare the results with those of model system 1 to investigate the effect of tether placement.

Figure 3(c) shows the phase diagram of model system 2 with a fixed volume fraction $(\phi=0.3)$ and varying tether length. This phase diagram shares the same overall structure as the phase diagram in figure 3(a), i.e., lamellar phases are observed for cubes with short tethers and cylindrical phases are observed for cubes with long tethers. However, the phase boundary between these two highly ordered phases is shifted towards the short tether end and the lamellae to cylinder ODT is observed for six-bead tethered cubes as opposed to the sevenand eight-bead tethered cubes in figure 3(a). This difference in phase behaviour can be explained by the increased steric repulsion of the tether beads due to the change in tether placement. As the two ends of the cyclic tether are moved further apart on the nanocube, the tether beads are forced to cover more surface area of the cube, further inhibiting the long range, flat face-to-face close packing required for the lamellar phase and thereby favouring the cylinder phase.

Figure 3(d) shows the phase diagram of model system 2 with a fixed tether length (eight beads) and varying volume fraction. This phase diagram also shares the same features with its counterpart discussed in section 3.1 , but with an increased micelle phase region, a decreased cylinder phase region, and a decreased lamellae phase region when comparing figure 3(d) with 3(b). This modification in phase behaviour is also caused by the stronger steric influence of repulsive tether beads induced by the change in tether placement. Increasing the distance between attachment points further inhibits the face-to-face cubic packing and stabilizes the micelle phase, as both cylinder and lamellar phases require more face-to-face close packing than the micelle phase.

\subsection{Nanocubes with two linear tethers attached to two cube corners along the same edge}

The third system we investigated is that of nanocubes with two linear tethers attached to two cube corners along the same edge (model system 3, as shown in figure 1(c)). We compare this system with model system 1 to investigate the effect of tether topology.

Figure 3(e) shows the phase diagram of model system 3 with a fixed volume fraction $(\phi=0.3)$ and varying tether length. This phase diagram is similar to the phase diagram of model system 1 discussed in figure 3(a), but the phase boundary between the lamellar and cylindrical phases is further shifted towards short tethers and the lamellae phase can only be observed for five-bead cyclic tethers. This difference demonstrates that replacing one cyclic tether as in model 1 with two linear tethers as in model system 3 increases the steric effects of the tethers. Compared with a cyclic tether, the two linear tethers have less restriction for those beads adjacent to the two free ends, allowing them to occupy more volume in good solvent, even penetrating into the cube-rich domain to further inhibit the long range, flat face-to-face cubic packing required by lamellar phases and favouring the cylindrical phase.

Figure 3(f) shows the phase diagram of model system 3 with a fixed combined tether length (eight beads, comprised of two tethers of four beads each) and varying volume fraction. This phase diagram is also similar to its counterpart discussed in section 3.1, but with a slightly decreased lamellar phase region $(\phi=0.35$ and above in figure 3(f), compared to $\phi=0.3$ and above in figure 3(b)) and significantly lower ODT temperatures between disordered and lamellar phases (for example, with $\phi=0.4$, the ODT temperature is between 1.6 and 1.5 in figure 3(f), as opposed to between 1.9 and 1.8 in figure $3(\mathrm{~b})$ ). As previously mentioned, the end beads of the two linear tethers have larger local free volume, frustrating the formation of the lamellar structure and requiring a lower ODT temperature to form ordered structures.

\subsection{Nanocubes with two cyclic tethers attached to the same face}

The fourth system we investigate contains nanocubes with two cyclic tethers attached to the same face (model system 4 as shown in figure 1(d)). We compare this system with model systems 1-3 and tetra-tethered nanocubes [26] to investigate the effects of both tether number and tethering topology.

Figure $3(\mathrm{~g})$ shows the phase diagram of model system 4 with a fixed volume fraction $(\phi=0.3)$ and varying tether length. Unlike the phase diagrams discussed in the above sections, cylindrical phases are the only ordered phases in this phase diagram and the ODT temperatures are relatively lower (all ODT temperatures are below 1.0 in figure 3(g) as opposed to $\sim 1.1$ in figure $3(\mathrm{a})$ ). Compared with model system 1 with a single cyclic tether, two cyclic tethers with the same number of combined tether beads occupy more space in the vicinity of the nanocubes. The tethers further interrupt the flat face-to-face close packing and entirely eliminate the lamellar phase. Furthermore, the two cyclic tethers can shield the entire nanocube face to which they are attached, and even extend to the four adjacent faces, with very little space left to accommodate the close packing of neighbouring nanocubes, resulting in cylinders of square cross-section where each nanocube has only one nearest neighbour in the lateral dimension (figure 2(c)). As these square cylinders are found only when two cyclic tethers or four linear tethers [26] are attached to the same face of the nanocubes and rectangularshaped cylinders are found in all other systems studied here, our simulation results suggest that the number of tether junction points on nanocubes can be manipulated to control the local geometry of the assembled nanostructures as well as the overall mesophases.

Figure 3(h) shows the phase diagram of model system 4 with a fixed combined tether length (eight beads, comprised of two tethers of four beads each) and varying volume fraction. Again, only cylinder phases are found in the ordered phase regime, as the two cyclic tethers cover a major fraction of the nanocube surface, precluding the formation of face-toface cubic packing required by lamellar phases. This phase diagram can be compared with the tetra-tethered POSS phase diagrams in figure 11 (a) of [26], where four linear tethers each with two beads are attached to the same face of a nanocube. 
The two phase diagrams share the same general features, with roughly the same phase boundary between micelle and cylinder phases, but different ODT temperatures (e.g., with $\phi=0.3$, the ODT temperature is roughly 0.9 in figure $3(\mathrm{~h})$ as opposed to $\sim 0.77$ in [26]). Similar to the phase boundary shift induced by the tethering topology difference between model systems 1 and 3 , the free end beads of the linear tethers in tetra-tethered nanocubes [26] penetrate and frustrate face-toface close packing and command a lower ODT temperature to form ordered phases.

\section{Conclusion}

Inspired by recent experimental work, we have used a simplified molecular model to perform stochastic molecular dynamic simulations on the self-assembly of cyclic tethered POSS nanocubes in selective solvent. We have identified the equilibrium structures resulting from the NBB self-assemblies and mapped phase diagrams with varying tether length, volume fraction and temperature. We have systematically explored the effects of tether topology, number and placement on the self-assembled structures and the phase diagrams. We find that the sizes of the lamellar phase regions decrease when the end beads of cyclic tethers are placed further apart on the nanocube surfaces, when one cyclic tether is replaced with two linear tethers of the same total length, or when the number of loops attached to the nanocubes increases. We also find that the phase boundaries between disordered and ordered phases shift towards the low temperature end when cyclic tethers are replaced with linear tethers.

Our minimal model captures the essential features of NBB architecture and interaction specificity of a broad class of tethered nanocubes, including, but not limited to, molecular POSS cubes. A coarse-grained interaction potential has recently been derived for mono-tethered POSS and was found to have the same general form as that used here [36], and thus we are confident that the general structures obtained here will not change due to quantitative, but not qualitative, changes in the interaction potentials. Our results suggest that the face-to-face close packing of nanocubes favours the highly ordered lamellar and cylindrical phases. We demonstrate that it is possible to exploit the steric influence of tethers on such packing patterns by manipulating the tether topology, number and placement, thereby conferring precise control over the self-assembled nanostructures and phase behaviour. Our study implies new opportunities in controlling tethered nanocube assemblies and provides novel avenues for 'bottomup' nanomaterials design.

\section{Acknowledgments}

Financial support for this work was provided by the National Science Foundation under grant No. DMR-0103399. We thank the University of Michigan Center for Advanced Computing for support of our computer clusters.

\section{References}

[1] Whitesides G M and Grzybowski B 2002 Science 2952418

[2] Glotzer S C 2004 Science 306419

[3] Glotzer S C, Solomon M J and Kotov N A 2004 AIChE J. 502978

[4] Baney R H, Itoh M, Sakakibara A and Suzuki T 1995 Chem. Rev. 951409

[5] Provatas A and Matisons J G 1997 Trends Polym. Sci. 5327

[6] Zhang C X, Babonneau F, Bonhomme C, Laine R M, Soles C L, Hristov H A and Yee A F 1998 J. Am. Chem. Soc. 1208380

[7] Pyun J, Matyjaszewski K, Wu J, Kim G M, Chun S B and Mather P T 2003 Polymer 442739

[8] Waddon A J, Zheng L, Farris R J and Coughlin E B 2002 Nano Lett. 21149

[9] Cardoen G and Coughlin E B 2004 Macromolecules 375123

[10] Zheng L, Hong S, Cardoen G, Burgaz E, Gido S P and Coughlin E B 2004 Macromolecules 378606

[11] Feher F J and Budzichowski T A 1995 Polyhedron 143239

[12] Li G, Wang L, Ni H and Pittman C U 2001 J. Inorg. Organomet. Polym. 11123

[13] Laine R M 2005 J. Mater. Chem. 153725

[14] Knischka R, Dietsche F, Hanselmann R, Frey H and Mülhaupt R 1999 Langmuir 154752

[15] Kim K-M, Keum D-K and Chujo Y 2003 Macromolecules 36867

[16] Leu C-M, Reddy G M, Wei K-H and Shu C-F 2003 Chem. Mater. 152261

[17] Sanchez C, Soler-Illia G, Ribot F, Lalot T, Mayer C R and Cabuil V 2001 Chem. Mater. 133061

[18] Phillips S H, Haddad T S and Tomczak S J 2004 Curr. Opin. Solid State Mater. Sci. 821

[19] Kannan R Y, Salacinski H J, Butler P E and Seifalian A M 2005 Acc. Chem. Res. 38879

[20] McCabe C, Glotzer S C, Kieffer J, Neurock M and Cummings P T 2004 J. Comput. Theor. Nanosci. 1265

[21] Striolo A, McCabe C and Cummings P T 2005 J. Phys. Chem. B 10914300

[22] Striolo A, McCabe C and Cummings P T 2005 Macromolecules $\mathbf{3 8} 8950$

[23] Ionescu T C, Qi F, McCabe C, Striolo A, Kieffer J and Cummings P T 2006 J. Phys. Chem. B 1102502

[24] Lamm M H, Chen T and Glotzer S C 2003 Nano Lett. 3989

[25] Sheng Y J, Lin W J and Chen W C 2004 J. Chem. Phys. 1219693

[26] Chan E R, Zhang X, Lee C-Y, Neurock M and Glotzer S C 2005 Macromolecules 386168

[27] Zhang X, Chan E R and Glotzer S C 2005 J. Chem. Phys. 123184718

[27a] Horsch M A, Zhang Z L, Iacovella C R and Glotzer S C 2004 J. Chem. Phys. 2111455

[28] Yang W-Y, Ahn J-H, Yoo Y-S, Oh N-K and Lee M 2005 Nat. Mater. 4399

[29] Auner N, Bats J W, Katsoulis D E, Suto M, Tecklenburg R E and Zank G A 2000 Chem. Mater. 123402

[30] Grest G S and Kremer K 1986 Phys. Rev. A 333628

[31] Glotzer S C, Horsch M A, Iacovella C R, Zhang Z L, Chan E R and Zhang X 2005 Curr. Opin. Colloid Interface Sci. 10287

[32] Zhang Z L, Horsch M A, Lamm M H and Glotzer S C 2003 Nano Lett. 31341

[33] van Gunsteren W F, Berendsen H J C and Rullmann J A C 1981 Mol. Phys. 4469

[34] Grest G S, Lacasse M D, Kremer K and Gupta A M 1996 J. Chem. Phys. 10510583

[35] Allen M P and Tildesley D J 1987 Computer Simulation of Liquids (Oxford: Clarendon)

[36] Chan E R, Striolo A, McCabe C M, Glotzer S C and Cummings $\mathrm{P} T$ in preparation 